\title{
Handling Qualities Evaluation of Pilot Tools for Spacecraft Docking in Earth Orbit
}

\author{
Karl D. Bilimoria, ${ }^{*}$ Eric Mueller, ${ }^{\dagger}$ and Chad Frost ${ }^{\ddagger}$ \\ NASA Ames Research Center, Moffett Field, CA, 94035
}

\begin{abstract}
A new generation of spacecraft is now under development by NASA to replace the Space Shuttle and return astronauts to the Moon. These spacecraft will have a manual control capability for several mission tasks, and the ease and precision with which pilots can execute these tasks will have an important effect on mission risk and training costs. This paper focuses on the handling qualities of a spacecraft based on dynamics similar to that of the Crew Exploration Vehicle, during the last segment of the docking task with a space station in low Earth orbit. A previous study established that handling qualities for this task degrade significantly as the level of translation-into-rotation coupling increases. The goal of this study is to evaluate the efficacy of various pilot aids designed to mitigate the handling qualities degradation caused by this coupling. Four pilot tools were evaluated: dead-band box/indicator, flight-path marker, translation guidance cues, and feed-forward control. Each of these pilot tools improved handling qualities, generally with greater improvements resulting from using these tools in combination. A key result of this study is that feedforward control effectively counteracts coupling effects, providing solid Level 1 handling qualities for the spacecraft configuration evaluated.
\end{abstract}

\section{Introduction}

$\mathrm{H}$ andling qualities are those characteristics of a flight vehicle that govern the ease and precision with which a pilot is able to perform a flying task. ${ }^{1}$ Several factors influence a pilot's perception of the handling qualities. These factors include the stability and control characteristics of the unaugmented vehicle, the control systems that enhance these characteristics, the inceptors (e.g., stick or throttle lever) used by the pilot to transmit control commands, and the cues that provide flight information to the pilot. Cues that assist the pilot in the execution of the flying task may be visual (the displays, instrumentation, guidance and out-the-window view) proprioceptive, or aural. The effects of the above factors on handling qualities have been studied in atmospheric flight vehicles for over seventy years. ${ }^{1-4}$ Reference standards for the handling qualities of both fixed-wing aircraft ${ }^{5}$ and rotary-wing aircraft ${ }^{6}$ have been developed, and are now in common use. Broadly speaking, these standards define a subset of the dynamics/control design space that provides good handling qualities for a given vehicle type and flying task. For example, the standards may specify a range of combinations of damping and natural frequency for a large aircraft during landing that corresponds with acceptable and unacceptable handling qualities.

At this time, no reference standards exist for handling qualities of piloted spacecraft. Handling qualities have been assessed for some space vehicles; $;^{7-9}$ however, the general objective of these studies was to evaluate and/or address deficiencies in the handling qualities of an existing point design for a specific vehicle. A more systematic approach would map out handling qualities variations over a broad range of design variables to determine desirable regions in the design space for a class of vehicles.

NASA and industry are designing a new generation of piloted spacecraft. ${ }^{10}$ These vehicles include the Crew Exploration Vehicle (CEV, also known as Orion) to replace the Space Shuttle and ferry astronauts to lunar orbit, and the Altair spacecraft to provide transportation between lunar orbit and the lunar surface. The ability of pilots to successfully carry out their missions will be determined in part by the handling qualities of these new spacecraft.

\footnotetext{
"Research Scientist, Flight Trajectory Dynamics and Controls Branch, Mail Stop 210-10, Karl.Bilimoria@nasa.gov. Associate Fellow, AIAA.

${ }^{\dagger}$ Aerospace Engineer, Flight Trajectory Dynamics and Controls Branch, Mail Stop 210-10, Eric.Mueller@nasa.gov. Member, AIAA.

${ }^{\ddagger}$ Deputy, Autonomous Systems and Robotics, Intelligent Systems Division, Mail Stop 269-1, Chad.R.Frost@nasa.gov. Associate Fellow, AIAA.
} 
Some operational tasks may be fully automated, while other tasks are executed with a human pilot fully engaged in the control loop. Even for the nominally automated tasks, a backup manual control capability is generally required so that a human pilot may take over when an automated system or critical sub-component of the spacecraft fails. In these cases of emergency reversion to manual control, where the pilot role abruptly switches from monitoring to active control, it is even more important that the vehicle have good handling qualities. It is, therefore, desirable for spacecraft designers to assess early in the design cycle what the handling qualities will likely be, and to adjust their design if necessary to ensure that adequate handling qualities are preserved even in degraded or failed operational modes.

An effort to develop design guidelines for spacecraft handling qualities was initiated by NASA in 2007 . A comprehensive set of guidelines should cover all classes of spacecraft and phases of flight; however, near-term NASA program goals make it necessary to focus initially on a few specific and relevant aspects. Preliminary studies of lunar landing ${ }^{11}$ and Earth orbit docking have been conducted. ${ }^{12,13}$ This paper reports a follow-on experiment investigating the effect of pilot tools on handling qualities for spacecraft docking in low Earth orbit; specifically, the attenuation of handling qualities degradation arising from translation-into-rotation coupling.

\section{Pilot Tools for Docking}

In current operations of Space Shuttle dockings with the International Space Station (ISS), an attitude alignment maneuver is performed to match the attitude of the Shuttle with that of the ISS and the final phase of docking is conducted with the Shuttle's attitude hold system engaged. The attitude control system fires the Shuttle's Reaction Control System (RCS) jets to create the roll/pitch/yaw moments necessary to hold its attitude within specified deadbands. The pilot uses a translation hand controller (THC) to make translational control inputs as necessary to null the position error between the center of the Shuttle docking port and the center of the ISS docking port. This generally requires pilot inputs along two translational axes: up/down inputs to make trajectory changes in the orbital plane, and left/right inputs to make trajectory changes perpendicular to the orbital plane. The pilot visually estimates the position error by utilizing a simple optical tool called a reticle, which provides cross-hairs indicating the centerline of the spacecraft's dock center overlaid on a view of the ISS dock. It is noted that for a successful docking, the position error limit is of the order of an inch $(2.54 \mathrm{~cm})$.

In general, the thrust lines of the RCS jets do not pass through the vehicle center of mass (c.m.), resulting in translation-into-rotation coupling. In a prior study ${ }^{12}$ of the Crew Exploration Vehicle (CEV) docking with the ISS, it was found that this coupling significantly degraded handling qualities. In the current study, pilot tools were developed with the goal of improving handling qualities in the presence of substantial translation-into-rotation coupling. These tools are described below.

\section{Dead-band Box}

A previous experiment ${ }^{12}$ revealed that a key source of pilot workload was determining when the attitude hold system would hit the dead-band and automatically fire RCS jets. Figure 1 shows a square box around the reticle cross-hairs. This attitude dead-band box indicates CEV pitch and yaw errors relative to the attitude-hold dead-band. The illustration in Fig. 1 indicates that the CEV is pitched down and yawed right.

Details will be provided in the final version of the paper.

\section{Flight-path Marker}

The aircraft-like symbol in Fig. 1 is a conventional representation of the flight-path marker. It indicates the projection of the CEV dock center's translational velocity relative to the ISS. The illustration in Fig. 1 indicates that if the current relative velocity persists, the CEV dock center will make contact below and to the right of the ISS dock centerline.

Details will be provided in the final version of the paper.

\section{Guidance Cues}

The number and directionality (right/left and up/down) of THC inputs required for a successful docking are indicated by red dots superimposed on the reticle. The illustration in Fig. 2 indicates that the pilot should make three THC pulses upwards and two pulses to the left. The guidance algorithm is a state feedback law that has two phases. The goal of the first phase is to take out the radial offset by zeroing out the radial position error and radial velocity before the dock-to-dock axial distance drops below $3 \mathrm{ft}$. The second phase provides fine tuning to compensate for drift.

Details will be provided in the final version of the paper. 


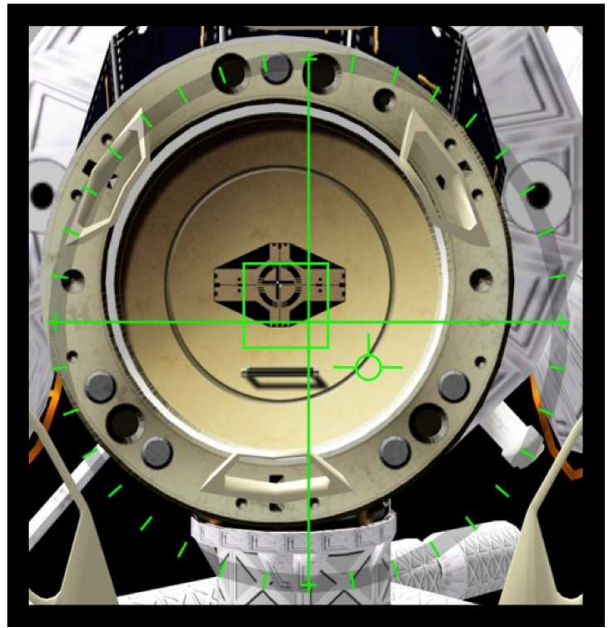

Figure 1. Dead-band box and flight-path marker

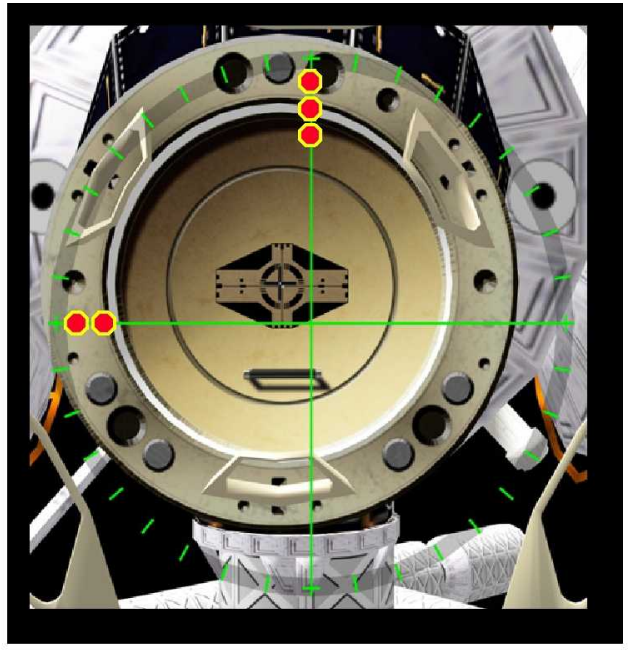

Figure 2. Translation guidance cues

\section{Feed-forward Attitude Control}

The standard type of attitude control employs feedback and is reactive in nature. For example, consider a THC input commanding a left-pointing force behind the vehicle c.m.; this force will create a nose-right yaw error due to translation-into-rotation coupling. In a feedback attitude hold control system, RCS jet firings to create a nose-left yawing moment are commanded only after the yaw error increases to a value that exceeds a specified dead-band.

A feed-forward control system is proactive in nature. In the example above, a feed-forward control system would first estimate the undesired nose-right yawing moment that would arise from a left THC input, and then fire RCS jets simultaneously to provide a left-pointing force as well as a nose-left yawing moment that cancels out the translation-into-rotation coupling effect. In practice, the coupling effect is not completely cancelled but is greatly attenuated. The operation of the feed-forward control system is transparent to the pilot; the vehicle responds as if it had very low translation-into-rotation coupling.

Details will be provided in the final version of the paper.

\section{Experiment Design}

The principal objective of this experiment was to evaluate the effect of various pilot tools on spacecraft handling qualities for Earth orbit docking. A dynamics and control model was implemented on the NASA Ames Vertical Motion Simulator (VMS), and a piloted evaluation of docking handling qualities was conducted in May-June 2008. The motion of both vehicles was modeled, including primary orbital mechanics effects, using the flight dynamics model described in Ref. 12. This section describes various aspects of the experiment design.

\section{Flying Task}

The task selected was the final stage of docking operations during which the visiting vehicle, in this case modeled on the Crew Exploration Vehicle (CEV), approached the International Space Station (ISS) along its velocity vector (this is known as the V-bar approach). In this experiment, the ISS was in a circular orbit $350 \mathrm{~km}$ above the surface of the Earth, and experienced no perturbations in position or attitude during the simulation run. At the start of the simulation run, the CEV was in essentially the same orbit as the ISS and was positioned slightly ahead of the ISS with the CEV's nose pointed along its negative velocity vector. The axial distance between the CEV and ISS docking ports was $10 \mathrm{ft}$ and the relative axial closing speed was $0.1 \mathrm{fps}$, resulting in a nominal run time of $100 \mathrm{sec}$.

In order to provide sufficient piloting challenge and expose any handling qualities issues, a radial offset error was applied to the initial position of the CEV dock relative to the ISS dock. This resulted in two piloting subtasks: (1) make coarse trajectory changes to align the centerline of the CEV dock with the centerline of the ISS dock, and (2) make fine trajectory changes as necessary to maintain the docks' alignment. 


\section{Test Matrix}

The primary objective of this experiment was to evaluate the effect of various pilot tools, individually and in selected combinations, on spacecraft handling qualities for Earth orbit docking. Secondary objectives were to evaluate the effects of attitude-hold dead-band size and the magnitude of initial position errors (nominal vs. offset approach) on handling qualities for Earth orbit docking. The test matrix is depicted in Table 1.

Table 1. Experiment matrix

\begin{tabular}{|c|c|c|c|c|c|c|c|c|}
\hline $\begin{array}{c}\text { Pilot } \\
\text { Tools* }\end{array}$ R & None & $\begin{array}{l}\text { Dead-band } \\
\text { Indicator } \\
\text { (DBI) }\end{array}$ & $\begin{array}{c}\text { Flight-path } \\
\text { Marker } \\
\text { (FPM) }\end{array}$ & $\begin{array}{c}\text { DBI } \\
+ \\
\text { FPM }\end{array}$ & $\begin{array}{c}\text { THC } \\
\text { Guidance }\end{array}$ & $\begin{array}{c}\text { THC } \\
\text { Guidance } \\
+ \text { DBI } \\
+ \text { FPM }\end{array}$ & $\begin{array}{l}\text { Feed- } \\
\text { forward } \\
\text { Attitude } \\
\text { Control }\end{array}$ & $\begin{array}{l}\text { Feed-forward } \\
\text { Attitude } \\
\text { Control } \\
\text { + DBI + FPM }\end{array}$ \\
\hline $\begin{array}{c}\text { Offset approach; } \\
0.25 \text { deg dead-band }\end{array}$ & & & & & & & & \\
\hline $\begin{array}{l}\text { Nominal approach; } \\
0.25 \text { deg dead-band }\end{array}$ & & & & & & & & \\
\hline $\begin{array}{l}\text { Nominal approach; } \\
0.5 \text { deg dead-band }\end{array}$ & & & & & & & & \\
\hline
\end{tabular}

${ }^{*}$ In addition to reticle, which is available for all pilot tool configurations

\section{Evaluation Pilots}

12 experienced test pilots provided data for this experiment; they had an average of 6,500 hours in a variety of fixed/rotary wing aircraft. Two were NASA pilots with decades of aircraft flight test/simulation experience. There were 10 current/retired NASA pilot astronauts from the Space Shuttle program; they had flown a total of 11 missions as Pilot and $\mathbf{8}$ missions as Commander. Many of the astronauts had performed actual spacecraft dockings, and most of them had received extensive simulator training on rendezvous/docking operations. Each pilot was available to the experimenters for about 8 hours, and this time constraint was incorporated into the experiment design.

\section{Training Procedures}

Upon arrival, pilots received a detailed briefing on the experiment background and objectives, flying task, control system, test matrix, and data collection procedures. Including discussion time with the experimenters, this session lasted approximately one hour. This was followed by a training and familiarization session (about one-hour) in the simulator cockpit, where pilots practiced the flying task for various representative configurations drawn from the test matrix, until they felt comfortable that most of the learning curve was behind them.

\section{Data Collection Procedures}

Each pilot encountered the various experiment configurations in a different sequence, and was not told the value of the attitude hold dead-band and whether the feed-forward control system was on. For each test configuration, the pilot flew two data collection runs with an option for a third run if necessary.

In handling qualities experiments, pilots are generally asked to make a composite assessment of the overall performance across all data collection runs for a test configuration. It is important to note that this assessment takes into account not just the quantitative evaluation of the end-point (e.g., docking contact) performance but also a qualitative evaluation of the manner in which the vehicle gets to the end-point. This overall assessment of desired, adequate, or inadequate performance is utilized for walking through the decision tree in the Cooper-Harper chart. ${ }^{1}$ Desired performance is necessary but not sufficient for Level 1 ratings, and adequate performance is necessary but not sufficient for Level 2 ratings. 
At the end of each run, relevant docking contact performance parameters (see Table 2) were displayed to the pilot and experimenters, with values colored green, yellow, and red according to performance limits for docking contact. The values of performance bounds for the parameters were derived from structural limits for the docking mechanism.

Table 2. Limits of docking contact performance parameters

\begin{tabular}{|c|c|c|}
\hline & Desired & Adequate \\
\hline Radial Misalignment & $1.5 \mathrm{in}$ & 3.2 in \\
\hline Angular Misalignment & $\begin{array}{l}\text { Roll: } \pm 1.5 \text { deg } \\
\text { Pitch/Yaw: } 1.5 \text { deg } \\
\text { (vector sum) }\end{array}$ & $\begin{array}{l}\text { Roll: } \pm 3.0 \text { deg } \\
\text { Pitch/Yaw: } 3 \text { deg } \\
\text { (vector sum) }\end{array}$ \\
\hline Axial Closure Rate & 0.075 to $0.125 \mathrm{fps}$ & $\begin{array}{c}0.05 \text { to } 0.075 \mathrm{fps} \\
-\mathrm{OR}- \\
0.125 \text { to } 0.15 \mathrm{fps}\end{array}$ \\
\hline $\begin{array}{c}\text { Radial Closure Rate } \\
\text { (combined translational } \\
\text { and rotational rates) }\end{array}$ & $0.075 \mathrm{fps}$ & $0.15 \mathrm{fps}$ \\
\hline Relative Angular Rate & $\begin{array}{c} \pm 0.075 \mathrm{deg} / \mathrm{sec} \\
\text { (each axis) }\end{array}$ & $\begin{array}{c} \pm 0.15 \mathrm{deg} / \mathrm{sec} \\
\text { (each axis) }\end{array}$ \\
\hline
\end{tabular}

After making a composite assessment of the overall performance across the data collection runs for a test configuration, pilots walked through the Cooper-Harper chart and assigned a handling qualities rating for that test configuration. Next, they assigned ratings for each of the six components of the NASA Task Load Index. ${ }^{15}$ These six components were: physical demand, mental demand, temporal demand, performance, effort, and frustration. The relative weighting of these six components for the docking task was determined by a pilot questionnaire at the end of the experiment. As appropriate, pilots also made qualitative comments about the test configuration they had just evaluated. All pilot comments were recorded on electronic media; the experimenters noted key points.

After all test configurations had been evaluated, there was a debrief session. The pilots were asked to fill out a one-page questionnaire designed to elicit high-level comments on cockpit displays, out-the-window displays, guidance cues, control response, and experiment design. This was followed by a discussion with the experimenters.

\section{Simulation Environment}

The experiment was conducted on the Vertical Motion Simulator (VMS) at NASA Ames Research Center. The VMS is a large motion base simulator ${ }^{18}$ that has been used for numerous handling qualities evaluations. ${ }^{16} \mathrm{~A}$ single pilot seat was installed in the center of the simulator cab, with a researcher/observer seat immediately aft of the pilot seat. The out-the-window view showed the ISS approximately as it would appear from the left (commander's) seat of the CEV. A three-axis translational hand controller (THC) was installed on the left side of the pilot seat. Although a three-axis rotational hand controller (RHC) was installed on the right side of the pilot seat, it was not utilized in this experiment consistent with current operating procedure in the final phase of docking. A schematic of the cockpit layout including the two control inceptors is shown in Fig. 3.

The panel in front of the pilot seat had three 6.5-inch color flat panel displays, the contents of which are shown in Fig. 4. The right panel displayed an Attitude Direction Indicator (ADI) and also included tapes showing range and range-rate of the CEV's docking port relative to the center of the ISS docking port. The center panel displayed a simulated view from a camera mounted on the centerline of the CEV dock, overlaid with a green reticle (crosshairs); other pilot tools were overlaid on this display as appropriate for the test configuration. The ISS dock is the beige ring with numerous holes and three petal-like objects in the center of this display. The left panel displayed color coded data of performance parameters at docking contact, such as radial offset error and relative angular rates. 


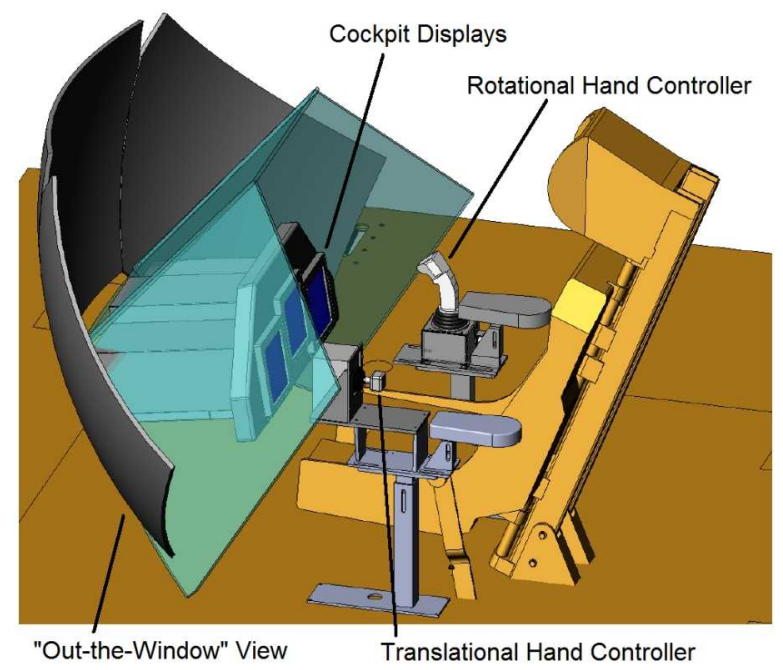

Figure 3. Simulator cockpit layout
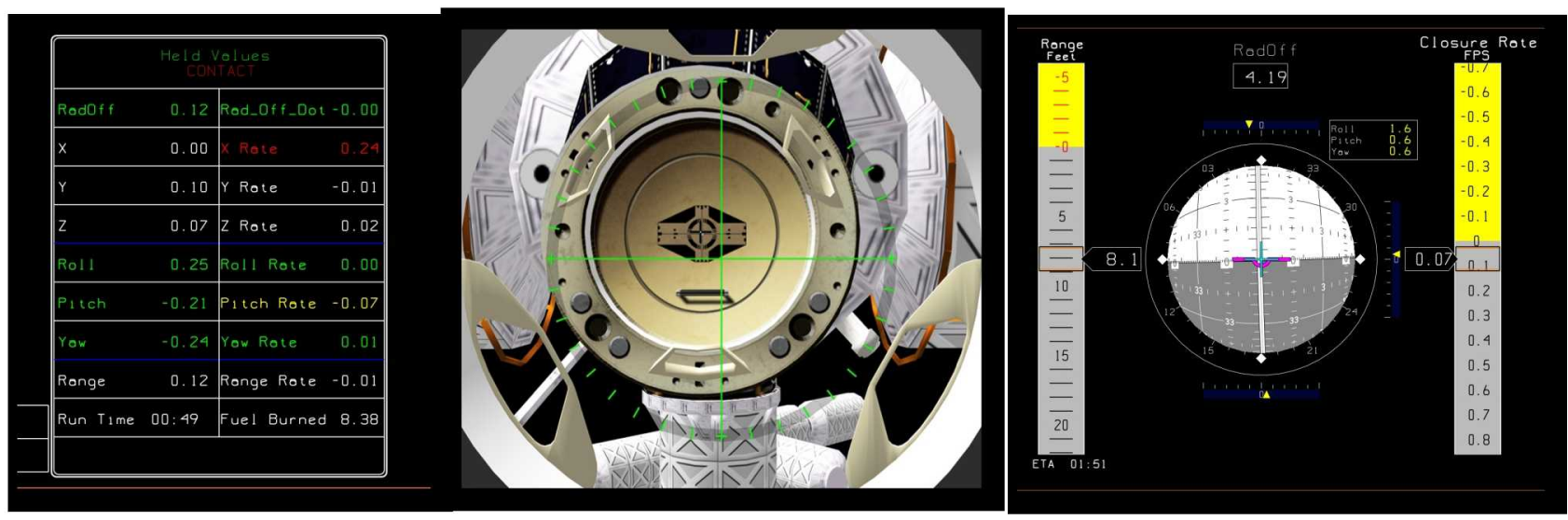

Figure 4. Simulator cockpit layout

Generic vehicle dynamics and control systems for translation and attitude were developed for this experiment using the most recent CEV configuration information available at the time. That design information included the Reaction Control System (RCS) thruster locations, vehicle dimensions, mass properties and other pertinent details, but did not include control system details. Control system designs representative of a range of possible implementations were developed for this experiment. A side view of the CEV showing the body axis coordinate system and important subsystems is provided Fig. 5.

The response type in the translation axes corresponded to a pulse mode. Displacement of the inceptor out of detent commands the appropriate RCS thrusters to fire for a specific duration, resulting in a fixed velocity increment (0.01 fps); the inceptor must be returned to detent before another command can be issued. This response type is similar to that used by the Space Shuttle today. It was also the highest rated response type in the handling qualities study reported in Ref. 12 .

The response type in the rotational axes was Rate Command / Attitude Hold (RCAH). Displacement of the inceptor out of detent commands the RCS thrusters to fire to achieve an angular rate proportional to the inceptor displacement; returning the inceptor to detent captures the spacecraft attitude, and fires thrusters as required to maintain that attitude within a specified dead-band. A phase-plane implementation based on the time-optimal (parabolic) switching curves were used to hold attitude (see Fig. 6). In that figure, the time-optimal switching curve 


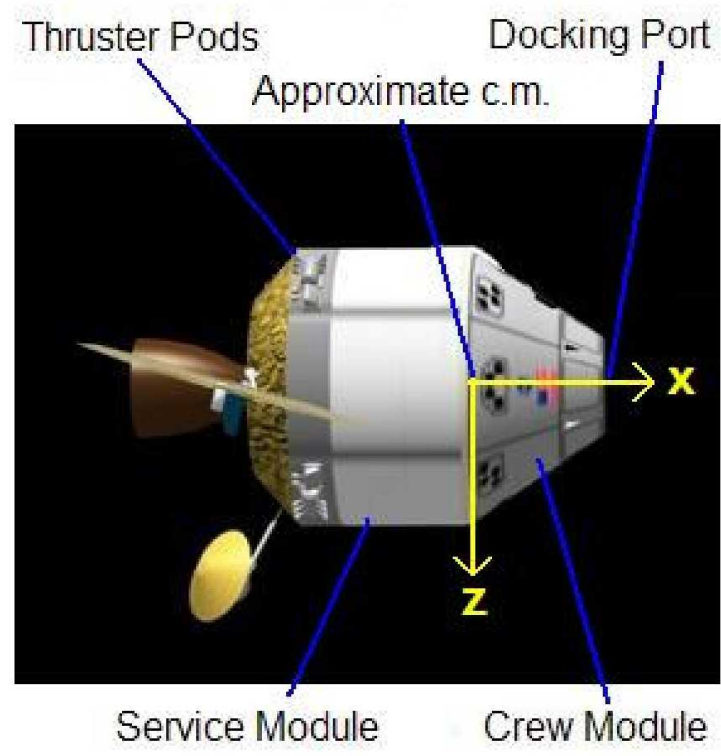

Figure 5. Schematic view of CEV.

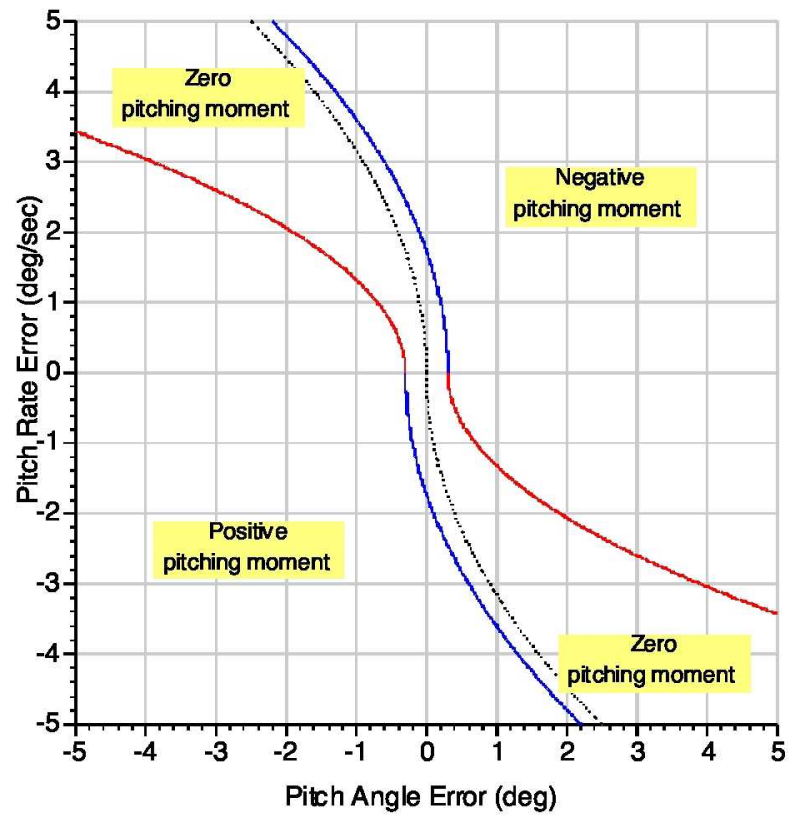

Figure 6. Switching curves (red and blue) for pitch attitude hold.

is shown as a dotted black line and the red and blue lines surrounding it represent the edges of the dead-band within which the RCS thrusters do not fire. The blue lines have the same shape as the time-optimal curve, but the red lines bend steeply towards the angular error axis in order to limit the angular rate at which the vehicle traverses the deadband. In essence, the degree of bending of the red lines represents a tradeoff between propellant use and the time it takes to remove errors.

\section{Results}

The data collection period in the VMS was May 27 through June 13, 2008. The 12 evaluation pilots provided Cooper-Harper ratings, NASA Task Load Index (TLX) ratings, and specific comments for each of the experiment configurations. They also provided feedback on the experiment design, as well as their overall impressions of the docking task. The performance parameters at docking contact were also recorded for each run.

A total of 617 data collection runs were made for this experiment. This section will discuss the qualitative and quantitative results obtained from these runs.

\section{Effect of pilot tools on handling qualities and workload ratings}

\section{Comparison across 0.25 and 0.5 deg attitude hold dead-bands for nominal approach}

Figure 7 presents color coded Cooper-Harper rating data for various pilot tools, individually and in selected combinations. It is noted that the reticle was present for all pilot tool configurations. For each pilot tool configuration, there are two data bars corresponding to 0.25 and 0.5 deg attitude hold dead-bands. As an example, the left-most bar in Fig. 7 indicates that for the test configuration of no tools and 0.25 deg dead-band, about $40 \%$ of the pilots gave a Level 1 rating, about $60 \%$ gave a Level 2 rating, and there were no Level 3 ratings.

It is evident that the larger $(0.5 \mathrm{deg})$ dead-band significantly degrades handling qualities, regardless of pilot tool configuration. For a fixed value of dead-band, the use of pilot tools improves handling qualities relative to the baseline of no tools (i.e., reticle only). The extent of improvement depends on the tool(s). It can be seen that the configuration of feed-forward control is the only one that received a Level 1 rating from all pilots and corresponds to the pilot tool configuration with the best handling qualities. 
Green, yellow, and red colors indicate Levels 1, 2, and 3 respectively; shades of color indicate the rating

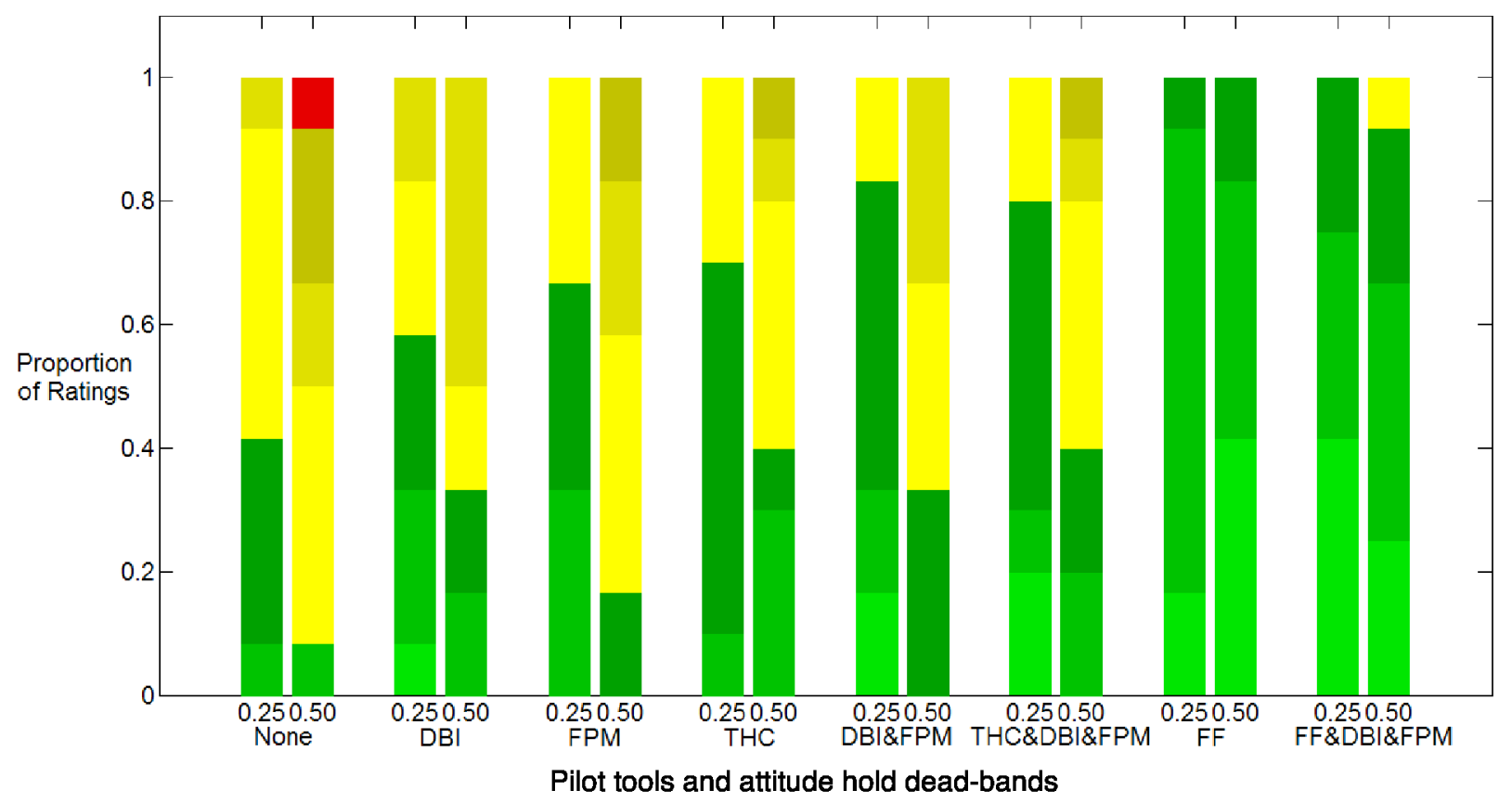

Figure 7. Cooper-Harper ratings for various pilot tools, 0.25 and 0.5 deg attitude hold dead-bands

Figure 8 shows workload (NASA Task Load Index) ratings for various pilot tools, individually and in selected combinations. It is noted that the reticle was present for all pilot tool configurations. For each pilot tool configuration, there are two data blocks corresponding to 0.25 and $0.5 \mathrm{deg}$ attitude hold dead-bands. The NASA TLX rating was computed as a weighted sum of the workload component ratings. The data in Fig. 8 is presented as a box-and-whiskers plot.

It is evident that the larger $(0.5 \mathrm{deg})$ dead-band significantly increases the pilot workload, for all tool configurations without feed-forward control. For a fixed value of dead-band, the use of pilot tools provides some reduction in workload relative to the baseline of no tools (i.e., reticle only). The extent of improvement depends on the tool(s). It is clear that feed-forward control provides a significant reduction in pilot workload.

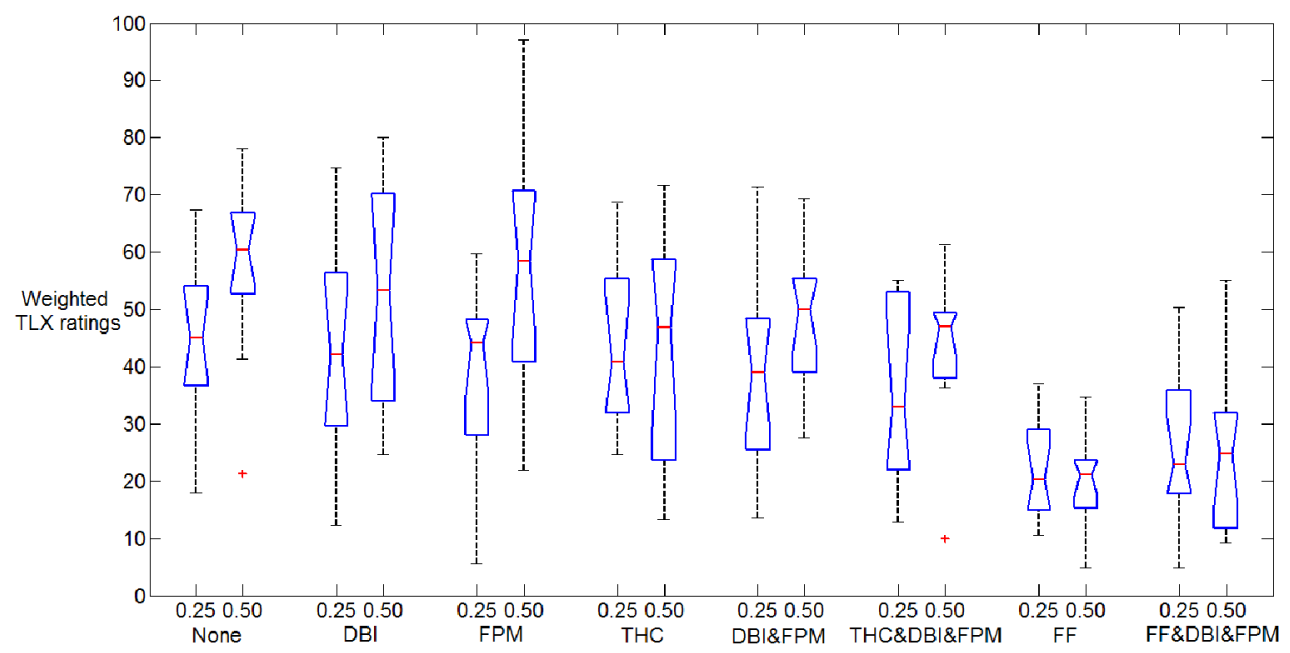

Pilot tools and attitude hold dead-bands

Figure 8. Workload ratings for various pilot tools, 0.25 and 0.5 deg attitude hold dead-bands 


\section{Comparison across nominal and offset approaches for 0.25 deg dead-band}

Figure 9 shows color coded Cooper-Harper rating data for various pilot tools, individually and in selected combinations. It is noted that the reticle was present for all pilot tool configurations. For each pilot tool configuration, there are two data bars corresponding to nominal and offset approaches. As an example, the left-most bar in Fig. 9 indicates that for the test configuration of no tools and offset approach, about $40 \%$ of the pilots gave a Level 1 rating, about $60 \%$ gave a Level 2 rating, and there were no Level 3 ratings.

Figure 9 shows that for both nominal and offset approaches, the use of pilot tools improves handling qualities relative to the baseline of no tools (i.e., reticle only). The extent of improvement depends on the tool(s). It can be seen that configurations of feed-forward control received a Level 1 rating from all pilots.

The docking task in this experiment had two piloting subtasks: (1) make coarse trajectory changes to align the centerline of the CEV dock with the centerline of the ISS dock, and (2) make fine trajectory changes as necessary to maintain the docks' alignment. The first sub-task was significant for the offset approach and negligible for the nominal approach, while the second subtask was essentially the same for both the offset and nominal approaches. Fig. 9 shows similar handling qualities for nominal and offset approaches, regardless of pilot tool configuration. This indicates that the second subtask, making fine trajectory changes to maintain alignment between the two docks, is the dominant one for the overall docking task.

Green, yellow, and red colors indicate Levels 1, 2, and 3 respectively; shades of color indicate the rating

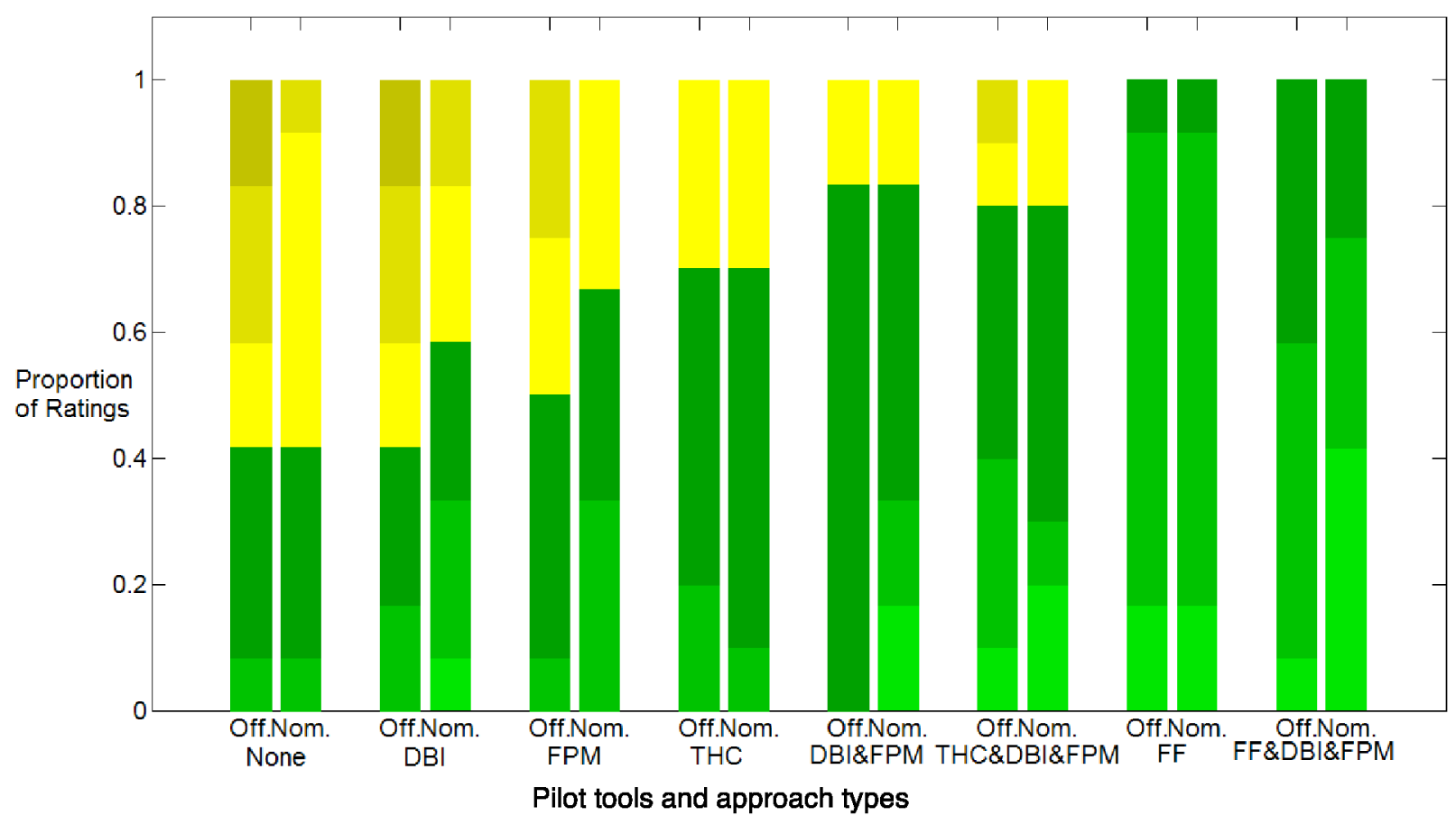

Figure 9. Cooper-Harper ratings for various pilot tools, nominal and offset approaches

Figure 10 shows workload (NASA Task Load Index) ratings for various pilot tools, individually and in selected combinations. It is noted that the reticle was present for all pilot tool configurations. For each pilot tool configuration, there are two data bars corresponding to nominal and offset approaches. The NASA TLX rating was computed as a weighted sum of the workload component ratings. The data in Fig. 10 is presented as a box-andwhiskers plot.

It is evident that pilot workload is significantly lower for tool configurations with feed-forward control. For a fixed value of dead-band, the use of pilot tools provides some reduction in workload relative to the baseline of no tools (i.e., reticle only). The extent of improvement depends on the tool(s). It is clear that feed-forward control provides a significant reduction in pilot workload. Fig. 10 shows similar workloads for nominal and offset approaches, regardless of pilot tool configuration. This indicates that the second piloting subtask, making fine trajectory changes to maintain alignment between the two docks, is the dominant one for the overall docking task. 


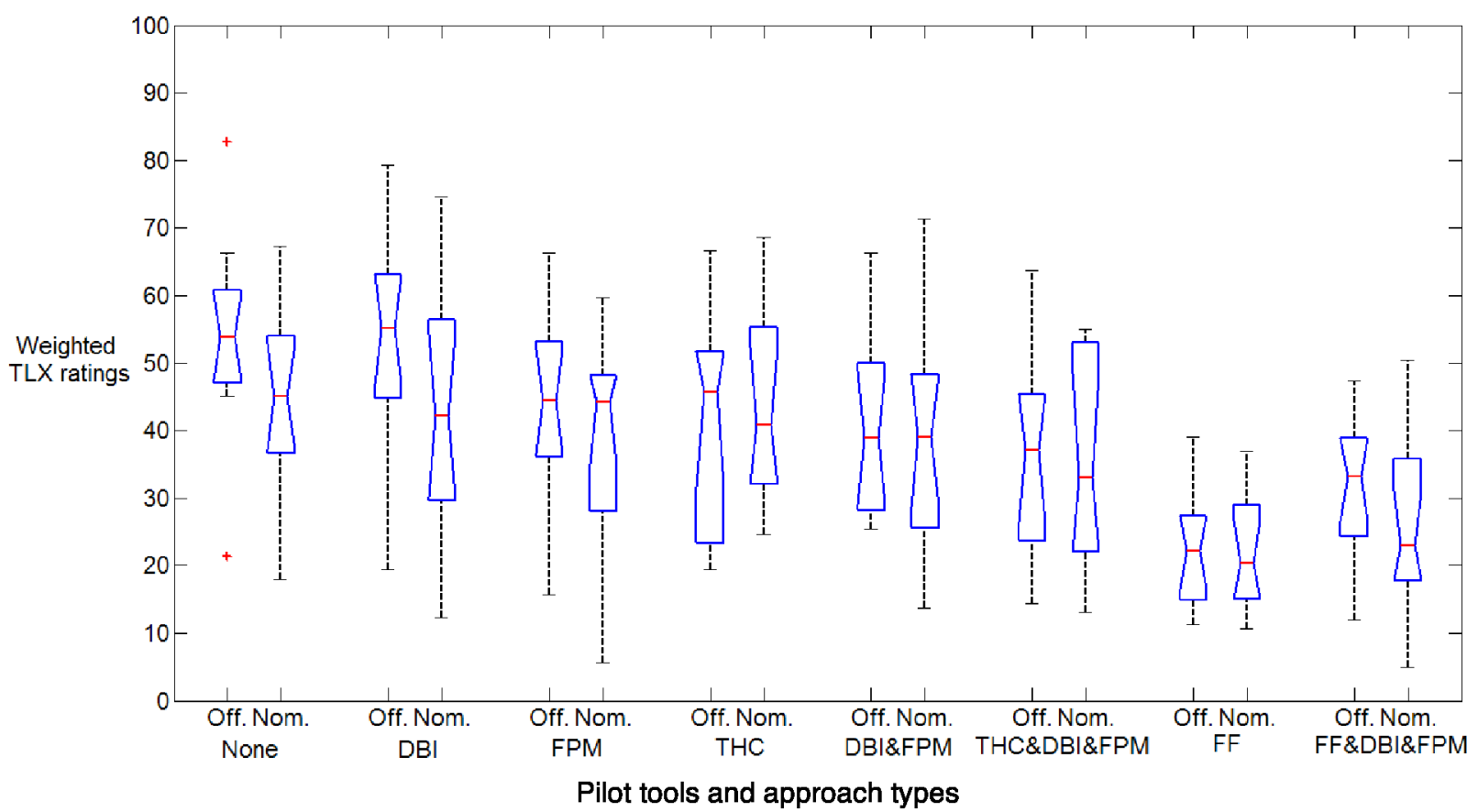

Figure 10. Workload ratings for various pilot tools, nominal and offset approaches

\section{Propellant usage}

Figure 11 shows the average propellant consumed per docking (nominal approach) for various pilot tools, individually and in selected combinations. It can be seen that the propellant usage with feed-forward control is similar to that of pilot tool configurations without feed-forward control, indicating that feed-forward control improves handling qualities without increasing propellant usage. Similarly, it can be seen that for each pilot tool configuration, there is no significant difference in fuel usage across the 0.25 and $0.5 \mathrm{deg}$ attitude hold dead-bands, indicating that the tighter dead-band $(0.25 \mathrm{deg})$ improves handling qualities without increasing propellant usage.

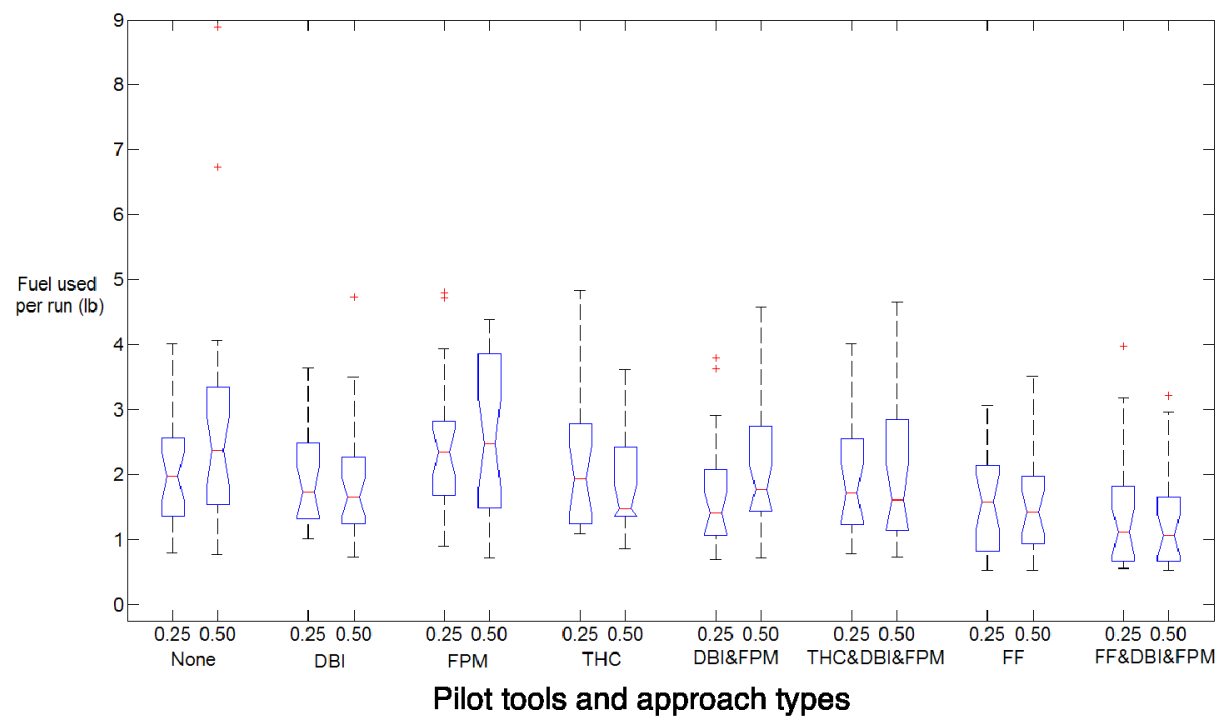

Figure 11. Propellant usage for various pilot tools, 0.25 and 0.5 deg attitude hold dead-bands 


\section{Conclusions}

An evaluation of handling qualities for a spacecraft docking in Earth orbit was conducted by 12 pilots, including 10 astronauts, flying the NASA Ames Vertical Motion Simulator. The objective was to study the effects of various pilot tools on handling qualities of a spacecraft approximating the Crew Exploration Vehicle, for the task of docking with the International Space Station. Four pilot tools were designed with the goal of enhancing handling qualities: dead-band box/indicator, flight-path marker, translation guidance cues, and feed-forward control. Handling qualities with these tools, individually and in selected combinations, were compared to a baseline no-tools configuration.

The baseline no-tools configuration had less than desirable handling qualities due to the effects of translationinto-rotation coupling; about $40 \%$ of the pilots gave it a Level 1 rating while the remainder (about $60 \%$ ) gave it a Level 2 rating. Use of dead-band box/indicator, flight-path marker, and translation guidance cues, individually and in selected combinations, improved handling qualities but did not make them solidly Level 1. Utilizing feedforward attitude control yielded a substantial improvement in handling qualities (virtually all ratings were Level 1) without affecting propellant usage.

For pilot tool configurations that did not utilize feed-forward control, it was found that a lower dead-band for attitude hold ( 0.25 deg vs. $0.5 \mathrm{deg})$ substantially improved handling qualities without affecting propellant usage. It was also found that the sub-task of making fine trajectory changes to maintain alignment between the two docks dominates the docking task.

\section{Acknowledgments}

The efforts of the SimLabs staff at NASA Ames are greatly appreciated. In particular, the authors would like to acknowledge the substantial contributions of Mike Weinstein who developed all software for the CEV dynamics/control model and also served as simulation engineer for the experiment. Bo Bobko served as project pilot and contributed to model development and testing. Boris Rabin created out-the-window views of the International Space Station. Steve Beard provided simulator cab graphics for Fig. 3.

Jim Dutton from NASA Johnson Space Center served as liaison with the Crew Office and provided valuable feedback during the development and testing phase of this effort.

\section{References}

${ }^{1}$ Cooper, G.E. and Harper, R.P., "The Use of Pilot Rating in the Evaluation of Aircraft Handling Qualities," NASA TN D-5153, April 1969.

${ }^{2}$ Soule, H.A., "Preliminary Investigation of the Flying Qualities of Airplanes," NACA Report 700, 1940.

${ }^{3}$ Gilruth, R.R., "Requirements for Satisfactory Flying Qualities of Airplanes," NACA TR 755, 1943.

${ }^{4}$ Cooper, G.E. and Harper, R.P., "Handling Qualities and Pilot Evaluation," Journal of Guidance, Control, and Dynamics, Vol. 9, No. 5, Sep-Oct 1986, pp. 515-529.

${ }^{5}$ Anon, Military Specification, Flying Qualities of Piloted Airplanes, MIL-F-8785C, Nov 1980.

6"'Aeronautical Design Standard, Performance Specification: Handling Qualities Requirements for Military Rotorcraft," US Army Aviation and Missile Command, ADS-33E-PRF, Mar. 2000.

${ }^{7}$ Cheatham, D.C. and Hackler, C.T., "Handling Qualities for Pilot Control of Apollo Lunar-Landing Spacecraft," Journal of Spacecraft, Vol. 3, No. 5, May 1966, pp. 632-638.

${ }^{8}$ Hackler C.T., Brickel, J.R., Smith, H.E., and Cheatham, D.C., "Lunar Module Pilot Control Considerations," NASA TN D-4131, February 1968.

9Powers, B.G., "Space Shuttle Longitudinal Landing Flying Qualities," Journal of Guidance, Control, and Dynamics, Vol. 9, No. 5, Sep-Oct 1986, pp. 566-572.

${ }^{10}$ National Aeronautics and Space Administration, "NASA's Exploration Systems Architecture Study," NASA TM-2005214062 , Nov 2005.

${ }^{11}$ Bilimoria, K.D., "Effects of Control Power and Guidance Cues on Lunar Lander Handling Qualities," Paper No. 20087799, AIAA Space 2008 Conference, San Diego, CA, September 2008.

${ }^{12}$ Mueller, E., Bilimoria, K.D., and Frost, C., "Handling Qualities Evaluation for Spacecraft Docking in Low Earth Orbit," Paper No. 2008-6832, AIAA Guidance, Navigation, and Control Conference, August 2008.

${ }^{13}$ Bailey, R., Jackson, B., Goodrich, K., Ragsdale, A., Neuhaus, J., Barnes, J., "Initial Investigation of Reaction Control System Design on Spacecraft Handling Qualities for Earth Orbit Docking," Paper No. 2008-6553, AIAA Atmospheric Flight Mechanics Conference, August, 2008.

${ }^{14}$ McFarland, R.E., "A Standard Kinematic Model for Flight Simulation at NASA-Ames," NASA CR-2497, January 1975. 
${ }^{15}$ Hart, S.G. and Staveland, L.E., "Development of NASA-TLX (Task Load Index): Results of Empirical and Theoretical Research," in Human Mental Workload, P.A. Hancock and N. Meshkati (Eds.), North Holland Press, Amsterdam, The Netherlands, 1988, pp. 239-350.

${ }^{16}$ Aponso, B.L., Tran, D.T., and Schroeder, J.A. "Rotorcraft Research at the NASA Vertical Motion Simulator," Proceedings of the $64^{\text {th }}$ Annual Forum of the American Helicopter Society, April 2008. 